\title{
Waterhammer effects in the case of air release
}

\author{
S. Mambretti \\ Wessex Institute of Technology, UK
}

\begin{abstract}
In the case of unsteady flow in pipes, the main problems seem to be the modelling of headlosses, especially when air release phenomena occur. A number of approaches are possible, but in this paper only one-dimensional and one-phase models have been investigated, in order to check their adequacy in reproducing real data, as they require much less computational effort and are therefore much faster and easy to apply.

Laboratory experiments have been performed at the Hydraulic Laboratory of the Politecnico di Milano, Italy, and simple models have been implemented in a computer code and then applied.

The results are reported and commented; it appears that to reach some achievements, some other points have to be discarded and to improve the overall solution it is probably necessary to use two-dimensional two-phase models.
\end{abstract} Keywords: waterhammer, column separation, air release, headlosses.

\section{Introduction}

There are a number of examples of benefits derived by the presence of unsteady flow, but in most of the cases this phenomenon has destructive characteristics. This is why the subject has been studied since the XIX Century. However, still many uncertainties are present when the model of a complex system is built.

One of the main problems is related to the use of steady flow formulas to compute the headlosses.

Generally speaking, smoothing and phase shift are present even when no air release occurs [1], implying the problems are tied to the unsteady flow phenomena; the reasons for such inadequacy have to be found [2] in the different velocity profiles between those in unsteady flow and those correspondingly (i.e. with the same discharge) in steady flow. In unsteady flow the gradient of the velocity close to the pipe wall is steeper than in steady flow and therefore the stresses are higher, and finally so are the resistances [3]. 
To evaluate the resistances in the case of unsteady flow a number of approaches are possible [4] which can be of increasing complexity, starting with the consideration of the variations of the average velocity to the most complex models which include second derivatives of the velocities both in the longitudinal and in the axial directions.

In particular, in the following the references will be related to the one dimensional IAB (Instantaneous Acceleration Based) [5-7] or MIAB models (Modified Instantaneous Acceleration Based) [8, 9].

The aim of this paper is to check whether the one dimensional models can be adapted to the cases when the presence of air is to be expected. To this end, laboratory experiments have been performed at the Hydraulic Laboratory of the Politecnico di Milano, Italy, and the mentioned models have been implemented in a computer code and then applied.

The results are reported and commented; it appears that to reach some achievements, some other points have to be discarded and to improve the overall solution it is probably necessary to use two-dimensional two-phase models.

\section{Experimental set-up and results}

In order to check the results carried out from the models, in the Hydraulic Laboratory of the Politecnico di Milano a simple plant has been built. In figure 1 a simple sketch of the plant is presented. The plant is constituted of a simple pipe which links two reservoirs, positioned at different levels. From the upstream reservoir a pipe starts with diameter $D_{1}=300 \mathrm{~mm}$ which reduces to $D_{2}=150 \mathrm{~mm}$ and finally to $D_{3}=52 \mathrm{~mm}$. Immediately downstream of the latter reduction a valve to regulate the discharge is positioned and after that there are the instruments to record experimental data.

The instrumentation is constituted by a magnetic flow discharge measurement, an electro-valve which closes extremely fast and that has been used to create the transient effects, and a pressure transducer, linked to the recording system which allows the data to be transferred to a personal computer.

The final part of the plant is constituted of an iron pipe with diameter $D_{3}=52$ $\mathrm{mm}$ gathered in a coil made of 26 spires for a total length of about $90 \mathrm{~m}$. This is finally linked to a downstream reservoir.

Pipe roughness has been measured during steady flow experiments and can be expressed with Manning parameter equal to $0.0091 \mathrm{~s} \cdot \mathrm{m}^{-1 / 3}$.

The pipe diameter is $D=52 \mathrm{~mm}$, the wall thickness is $e=5 \mathrm{~mm}$, the bulk elasticity modulus for iron is $E=2.0 \cdot 10^{11} \mathrm{~N} / \mathrm{m}^{2}$ and the water elasticity is $\varepsilon=2.14 \cdot 10^{9} \mathrm{~N} / \mathrm{m}^{2}$ while water density is $\rho=1000 \mathrm{~kg} / \mathrm{m}^{3}$. The wave celerity can be computed with the well known formula:

$$
c=\frac{\sqrt{\varepsilon / \rho}}{\sqrt{1+\frac{D \cdot \varepsilon}{e \cdot E}}}=\frac{\sqrt{2.14 \cdot 10^{9} / 1000}}{\sqrt{1+\frac{52 \cdot 2.14 \cdot 10^{9}}{5.0 \cdot 2.0 \cdot 10^{11}}}}=\frac{1462.87}{\sqrt{1.11}} \cong 1388 \mathrm{~m} / \mathrm{s}
$$




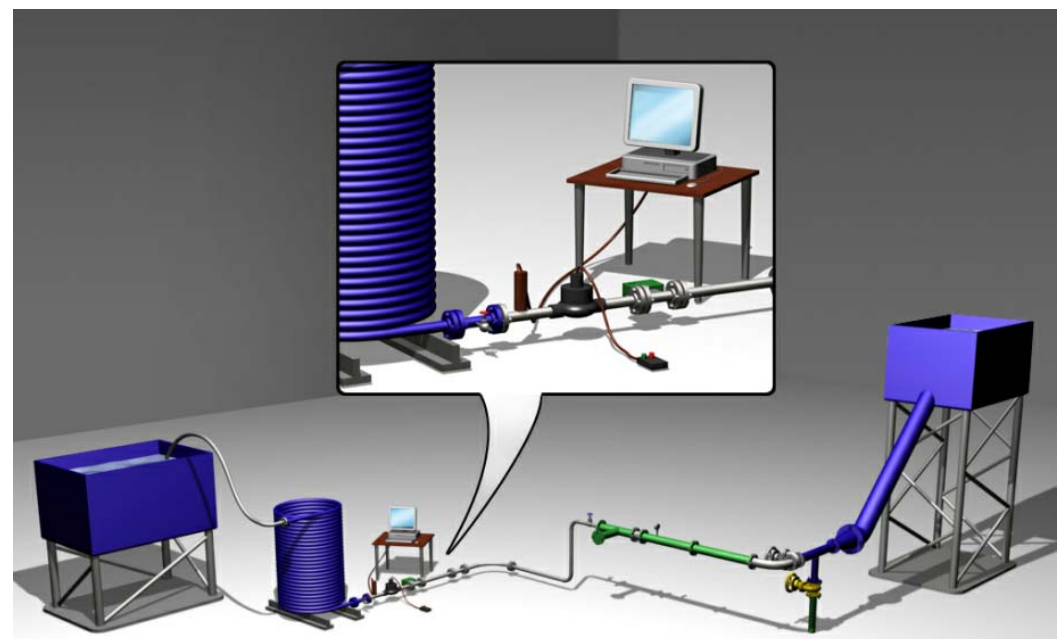

Figure 1: Sketch of the experimental plant built in the Politecnico di Milano.

Tests have been performed with five different discharges; test main characteristics are reported in table 1 . Some of the tests have been repeated in order to check the repeatability of the experiments, and the results showed they match perfectly.

Table 1: $\quad$ Main characteristics of the carried out tests.

\begin{tabular}{cccc}
\hline Test n. & $\begin{array}{c}\text { Discharge } \\
{[l / s]}\end{array}$ & $\begin{array}{c}\text { Velocity } \\
{[\mathrm{m} / \mathrm{s}]}\end{array}$ & $\begin{array}{c}\text { Upstream Head } \\
{[\mathrm{m}]}\end{array}$ \\
\hline 1 & 0.112 & 0.0527 & 5 \\
2 & 0.228 & 0.1074 & 5 \\
3 & 2.5 & 1.18 & 8.1 \\
4 & 3.0 & 1.41 & 9.7 \\
5 & 3.3 & 1.55 & 10.5 \\
\hline
\end{tabular}

It can be seen that for tests nn. 1 and 2 the pressure went below atmospheric; for tests nn. 3, 4 and 5 the pressure went to zero absolute and therefore water column separation has been obtained, for longer when initial velocity is higher. However, even in the first two tests air release was probable, as the recorded wave has been found slightly slower than computed, probably because of the air bubbles that increase liquid compressibility. 


\section{Numerical model}

Governing equations are the usual continuity (2) and momentum (3) equations, where the unknowns are the values of the head $h$ and velocity $V$.

$$
\begin{gathered}
\frac{\partial h}{\partial t}+V \cdot \frac{\partial h}{\partial x}+\frac{c^{2}}{g} \cdot \frac{\partial V}{\partial x}=0 \\
\frac{\partial V}{\partial t}+g \cdot \frac{\partial h}{\partial x}+V \frac{\partial V}{\partial x}+J \cdot g=0
\end{gathered}
$$

This system in compact form can be expressed as:

$$
\frac{\partial \vec{u}}{\partial t}+A(u) \cdot \frac{\partial \vec{u}}{\partial x}=\vec{E}
$$

where:

$$
u=\left|\begin{array}{l}
h \\
V
\end{array}\right| \quad A(u)=\left|\begin{array}{cc}
V & c^{2} / g \\
g & V
\end{array}\right| \quad E=\left|\begin{array}{c}
0 \\
-g \cdot J
\end{array}\right|
$$

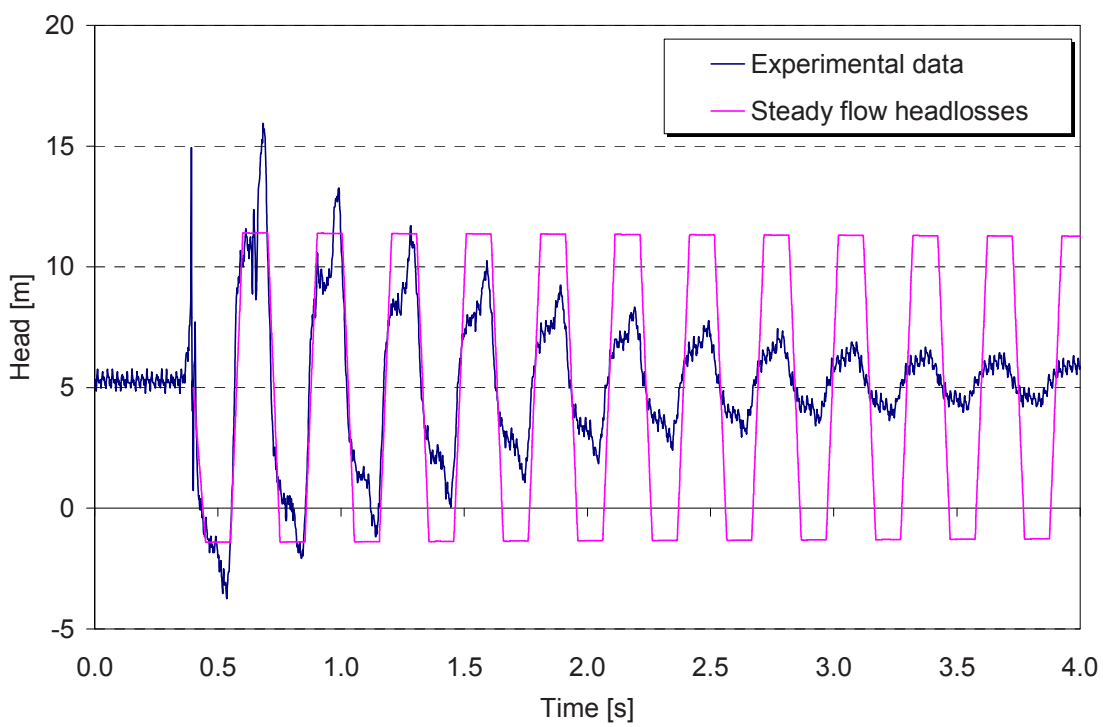

Figure 2: $\quad$ Test n. 1: Experimental data $V S$ model results, using steady flow formulation for headlosses. 
The numerical solution has been computed with the well-known LaxWendroff method [10], an explicit method which has been proved to be good for the solution of systems on hyperbolic linear differential equations, when the Courant-Friedrichs-Levy condition is satisfied.

In this case the governing equations carry a system which is not linear, but still the method can be applied with extensions given again by [10] or with the wider used formulation reported in [11], which is:

$$
u_{j}^{n+1}=u_{j}^{n}-A_{j}^{n} \cdot \frac{\lambda}{2}\left(u_{j+1}^{n}-u_{j-1}^{n}\right)+\frac{\lambda^{2}}{2} \cdot\left(A_{j}^{n}\right)^{2} \cdot\left(u_{j+1}^{n}-2 u_{j}^{n}+u_{j-1}^{n}\right)+\Delta t \cdot E_{j}^{n}
$$

As can be seen in figure 2, the steady flow headlosses are not appropriate to model the unsteady flow.

Brunone and Greco [5] proposed to compute the headlosses with the formula:

$$
J=J_{S}+J_{U}
$$

where $J_{S}$ is the usual Chézy resistance term, which is written as:

$$
J_{p}=\lambda \cdot \frac{V \cdot|V|}{2 \cdot g \cdot D}
$$

and $J_{U}$ is a term related to the unsteadiness of the flow, and the Authors expressed it as:

$$
J_{U}=k_{1} \cdot \frac{\partial Q}{\partial t}
$$

being $k_{1}$ an appropriate constant for smoothing and which is zero when:

$$
\operatorname{sign} Q \neq \operatorname{sign} \partial Q / \partial t
$$

While the formal expression for the solution is still (4), the (5) change as follows:

$$
\begin{aligned}
& u=\left|\begin{array}{l}
h \\
V
\end{array}\right| \quad A(u)=\left|\begin{array}{cc}
V & \rho \cdot c^{2} \\
g /\left(1+k_{1} \cdot A_{S} \cdot g\right) & V /\left(1+k_{1} \cdot A_{S} \cdot g\right)
\end{array}\right| \\
& E=\left|-g /\left(1+k_{1} \cdot A_{S} \cdot g\right) \cdot J_{S}\right|
\end{aligned}
$$

where $A_{S}$ is the cross sectional area of the pipe. 
This model does not claim to give the exact solution at the problem, but simply introduces a new term for unsteady resistances. The choice to set to zero the constant $k_{1}$ is necessary in order avoid unphysical smaller headlosses. As described in [5] and reported in the following paragraphs, this model not only smoothes the oscillations, but also create undesired changes in the wave celerity and therefore creates phase shifts.

A more complex expression for evaluating the resistance can be found in [8], where it is reported the following:

$$
J=J_{S}+\frac{k_{2}}{g} \frac{\partial V}{\partial t}+\operatorname{sgn}\left(V \frac{\partial V}{\partial x}\right) \cdot \frac{k_{2} \cdot c}{g} \cdot \frac{\partial V}{\partial x}
$$

being $k_{2}$ an appropriate constant, obviously different from $k_{1}$.In [12] is reported that the smoothing term for unsteady flow is negligible when the flow is decelerating while in the accelerating phase $k_{3}$ is constant. This model is theoretically very interesting as it allows the separation between local and convective accelerations.

In [13] a new expression has been introduced, considering $k_{3}$ as variable in space and time. Theoretically, this approach allows a better modeling of the reality but, as it has been generally recognized, the analytical relationships or the graphs which allow the estimation of this parameter, have not general application when a one dimensional model is applied and therefore practical applications are difficult; therefore, in this paper $k_{3}$ is let constant.

However, the real key for modelling appropriately the real world is the constant (or variable) $k$. To this end, much effort has been devoted to the correct evaluation of this value. In $[6,7]$ a value is proposed for any situation, while in $[4,14,15]$ a range is given. In [8] a diagram is drawn in order to select the best value of this parameter depending on the flow conditions and [16] provides analytical formulations.

In many cases there is the request to know in advance at least some characteristics of the real response of the structure, but this is not the point of this research, which tries to check whether such formulations are appropriate.

\section{Tests with no column separation (tests 1 and 2)}

As can be seen in figure 3 , the application of a simple unsteady flow headlosses model as that of described in [5] and above reported with the formulas (7)-(10) produces very good results. The value of the smoothing parameter $k_{1}$ has been set equal to 0.0634 , after a brief calibration. Wave celerity has been let equal to $1220 \mathrm{~m} / \mathrm{s}$, as it was estimated from recorded data.

As can be observed in the figure, there is a slight phase shift because, as mentioned and can be demonstrated, the wave celerity is influenced by the insertion of that new term. This might imply some differences when the model is applied in a complex plant, as the waves do not combine properly, even if this is probably a minor problem. 
What is more important is that the maxima values of recorded pressures are slightly higher than those modelled. As known, these maxima values can be easily computed with the Allievi-Joukowsky formula (e.g. [17]) and the carried out values (6.42 $\mathrm{m}$ for test 1 and $12.8 \mathrm{~m}$ for test 2 ) perfectly match the model results. In other words, the experimental conditions are someway different from the theoretical conditions assumed in the models.

As can be seen, even if column separation has not been reached in these tests, pressure drop under atmospheric value and therefore probably air is released and formed bubbles. That would explain the asymmetric trends of the pressures, the different celerity of the wave and maybe the higher pressure values, which can be obtained when the bubbles collapse due to the high pressure.

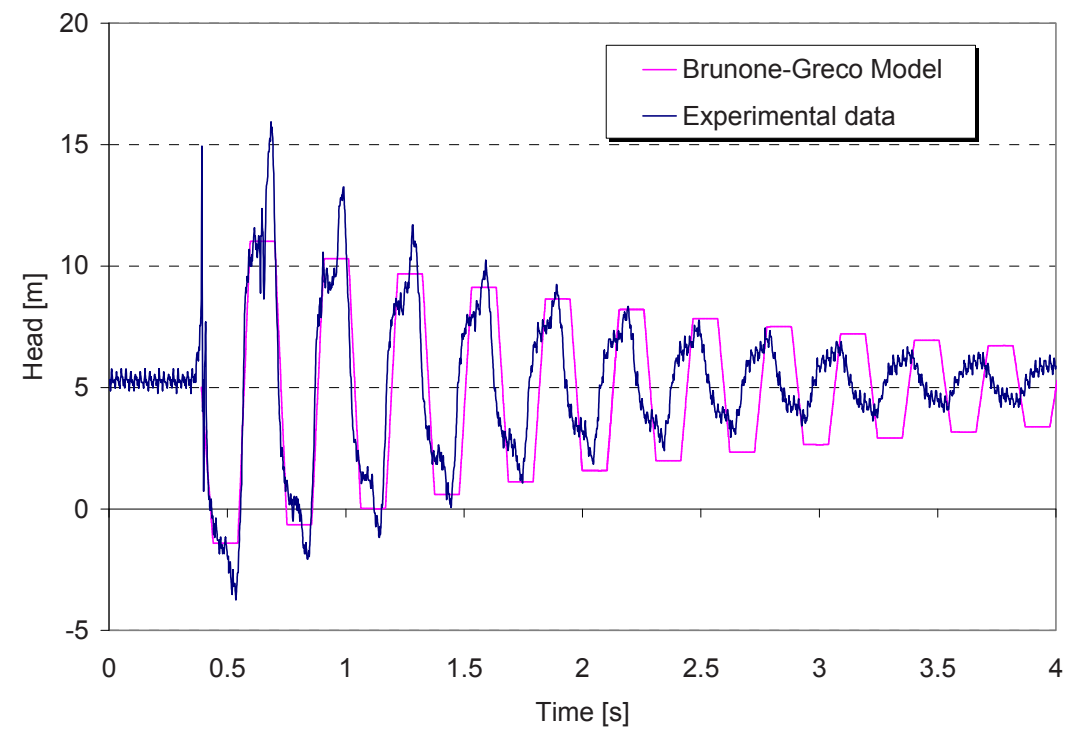

Figure 3: $\quad$ Test n. 1: Experimental data $V s$ model results, using Brunone and Greco [5] unsteady flow headlosses formula.

\section{Tests with water column separation (tests 3, 4 and 5)}

When the pressure drops down and gets close to the values of zero absolute (that means $p=-1 \mathrm{~atm}$ if referring to atmospheric pressure), the air nuclei dimensions increase and the flow becomes multiphase, which characteristics are determined by the resistance forces and by the slope of the pipe. The term "water column separation", therefore, does not necessarily imply the flow interruption, as the term would suggest [18]. 


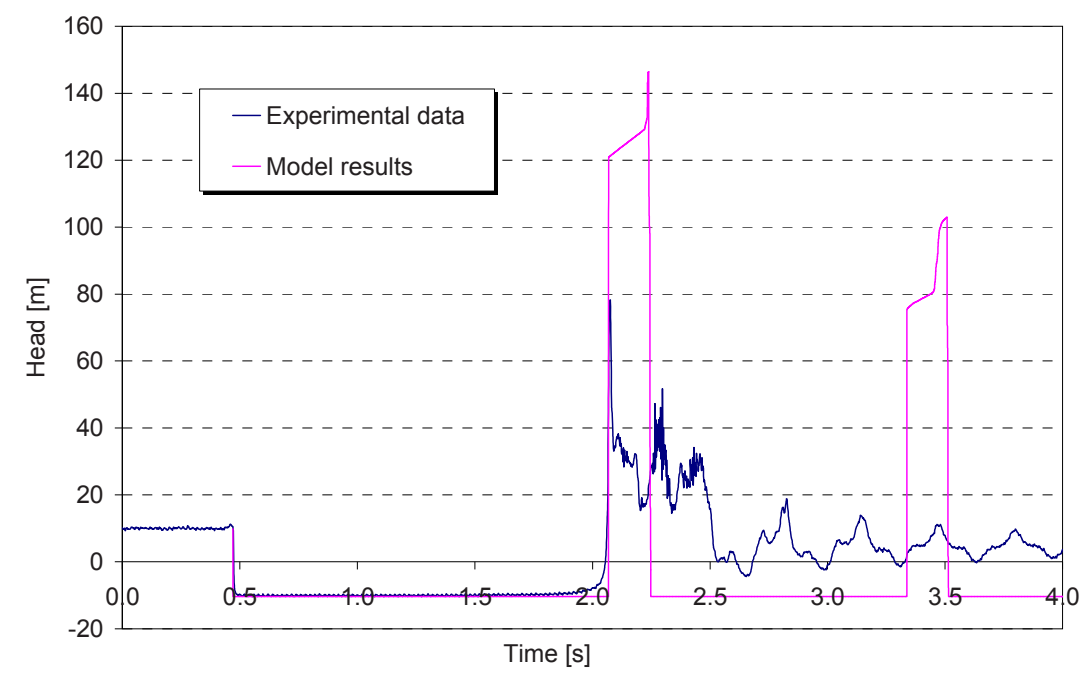

Figure 4: $\quad$ Test n. 3: Experimental data $V S$ model results, using steady flow formulation for headlosses and a simple model for water column separation.

However, the easiest way to model water column separation is considering the flow is actually separated in two halves. As boundary condition, in the section where cavitation occurs, the head is imposed and put equal to $p=$ $-1 \mathrm{~atm}$ which means $h=-10.33 \mathrm{~m}$. From the moment the pressure drops down to this value, this value is kept constant and the velocity of the column is computed as unknown parameter from the governing equations. In this way it is possible to compute the water column velocity and therefore its distance from the initial section and finally the time when the water column reattaches.

This very simple model performs pretty well if we are only interested in the estimation of the time of water column separation, as can be seen in figure 4 . However, the maximum computed pressure value is much higher than recorded; moreover, after the column reattaches there is a strong turbulence which reduces the head of the flow, which shows very small pressure oscillations that fade very quickly. On the contrary, the energy the flow has in the model is enough to bring to a new water column separation, which in the real world does not happen.

Due to the expectedly poor results obtained with the steady flow resistance formula, the model described in [8] has been used, reported in equation (12), with different values of the parameter $k_{3}$. As can be seen in figure 5 , no big improvements can be reached even with that model and even when similar (or larger) values of the $k_{3}$ constant are used. This is because in this case the main resistances are not due to the turbulent stresses, but to the presence of the bubbles [19]. Moreover, increasing the constant $k_{3}$ produces an increased phase shift bringing eventually to results which do not approximate in any way the recorded data. 


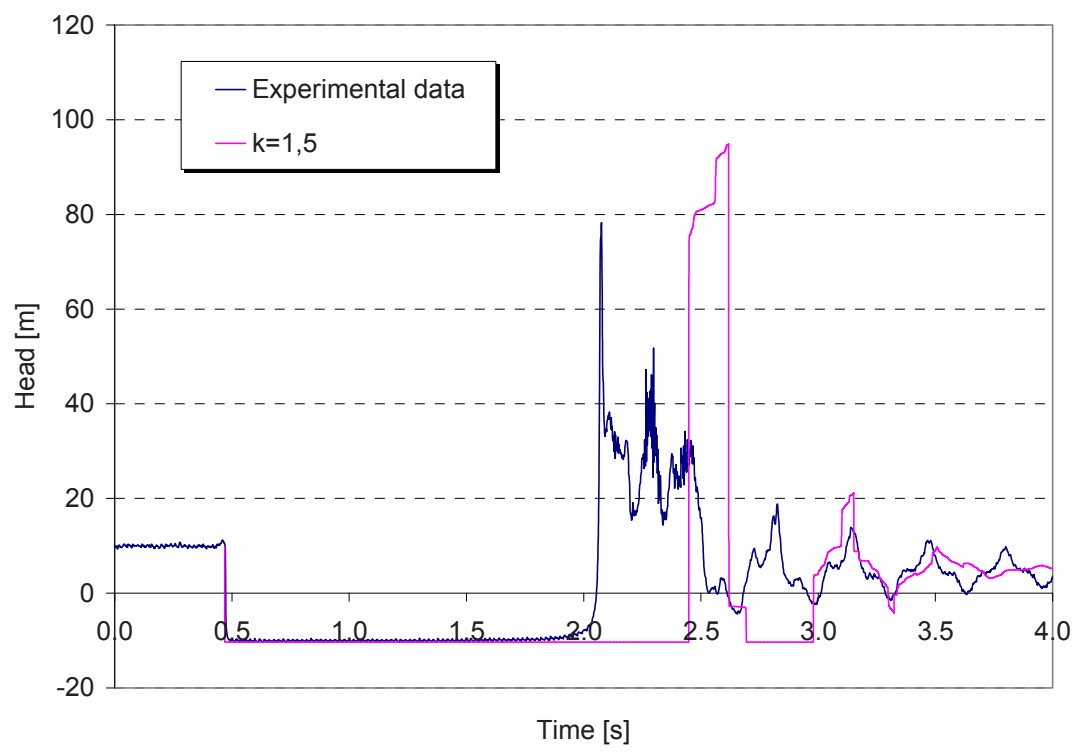

Figure 5: $\quad$ Test n. 3: Experimental data $V S$ model results, using unsteady flow formulation for headlosses and a simple model for water column separation.

\section{Concluding remarks}

In the case of unsteady flow in pipes, the main problems seem to be the modelling of headlosses, especially when air release phenomena occur. A number of approaches is possible, but in the paper the investigated models were only one-dimensional and one-phase, in order to check their adequacy in reproducing real data, even if after calibration, because they require much less computational effort and are therefore much faster and easy to be applied.

Laboratory experiments have been performed at the Hydraulic Laboratory of the Politecnico di Milano, Italy, and simple models have been implemented in a computer code and applied.

Results show, when simple air release due to the low pressures is to be feared, the mentioned models are able to reproduce at least the main features of the phenomenon. On the contrary, when water column separation occurs, these models fail to provide adequate response. In these cases, to improve the solution it is probably necessary to use two-dimensional and two-phase models.

In the following of the research, tests on these more complex models will be performed, in order to check their adequateness and whether it is possible to find an appropriate parameter to decide when to "switch" from the simpler, and faster, one-dimensional and one-phase models to the more complex ones. 


\section{References}

[1] Vardy A.E., "Unsteady Flows: Fact and friction" Proceeding of the 3rd International Conference on Pressure Surges, BHRA Fluid Engineering, Canterbury, England, A2, pp. 15-26, 1980.

[2] Van De Sande E. Belde A.P., Hamer B.J.G, Hiemistra W., "Velocity profiles in accelerating pipe flows started from rest", Proceeding of the 3rd International Conference on Pressure Surges, BHRA Fluid Engineering, Canterbury, England, A1, pp. 1-14, 1980.

[3] Cocchi G., "Esperimento sulla Resistenza al Deflusso con Moto Vario in Tubo", Atti dell'accademia delle scienze dell'istituto di Bologna, Classe di Scienze Fisiche, anno 276, Rendiconti, Serie XIV, tomo V, 1987-1988. (in Italian)

[4] Bergant A., Simpson A. R., "Estimating unsteady friction intransient cavitating pipe flow", Proc. 2nd Int. Conf. on Water Pipeline Systems, BHR Group, Edinburgh, Scotland, pp. 3-16, 1994.

[5] Brunone B., Greco M., "Un modello per la ricostruzione di fenomeni di colpo d'ariete anche in presenza di cavitazione", Riscontro sperimentale, 22nd Convegno di Nazionale di Idraulica e costruzioni Idrauliche, Cosenza, Italy, vol. 4, pp. 114-160, 1990 (in Italian).

[6] Brunone B., Golia U.M., Greco M., "Modelling of fast transient by numerical methods", Int. Meeting on Hydr. Transient and Water Column separation, E. Cabrera and M.A. Fanelli (eds.), IAHR, Valencia, Spain pp. 273-282, 1991.

[7] Brunone B., Golia U.M., Greco M., "Some remarks on the momentum equation for the fast transient", Int. Meeting on Hydr. Transient and Water Column separation, E. Cabrera and M.A. Fanelli (eds.), IAHR, Valencia, Spain pp. 201-205, 1991.

[8] Pezzinga G., "Evaluation of Unsteady Flow Resistances by Quasi-2D or 1D Models", Journal of Hydraulic Engineering, ASCE, vol. 126, n. 10, pp. 778-785, 2000.

[9] Ramos H., Covas D., Borga A., Loureiro D., "Surge damping analysis in pipe systems: modelling and experiments", Jour. Hydraulic Research, vol. 42, n. 4, pp. 413-425, 2004.

[10] Lax P.D., Wendroff B., "System of conservation laws", Comm. Pure and Applied Mathematics, vol. 13, pp. 217-237, 1960.

[11] Roe P.L., "Approximate Riemann Solvers, Parameter Vectors and Difference Schemes", J. Comput. Phys, vol. 43, pp. 357-372, 1981.

[12] Vitkovsky J.P., Bergant A., Simpson A.R., Lambert M.F., "Systematic Evaluation of One-Dimensional Unsteady Friction Models in Simple Pipelines", Journal of Hydraulic Engineering, vol. 132, n.7, pp. 696-708, 2006.

[13] Bouazza Z., Brunelle P.E., "A new friction model for transient pipe flows: Development of the frequency dependence approach of Brunone", Proc. Of the 9th Int. Conf. on Pressure Surges, BHR Group, Chester, U.K., pp. 391-404, 2004. 
[14] Bughazem M. B., Anderson A., "Problems with simple models for damping in unsteady flow", Proc., 7th Int. Conf. on Pressure Surges and Fluid Transients in Pipelines and Open Channels, BHR Group, Harrogate, U.K., pp. 537-548, 1996.

[15] Wylie, E. B., "Frictional effects in unsteady turbulent pipe flow.", Applied mechanics in the Americas, M. Rysz, L. A. Godoy, and L. E. Suarez, eds., vol. 5, The University of Iowa Press, Iowa City, Iowa, 5, pp. 29-34, 1996.

[16] Vardy A.E., Brown J.M.B., "On turbulent, un steady, smooth-pipe flow", Int. Conf. On Pressure Surges and Fluid Transients, BHR Group, Harrogate, England, pp. 289-311, 1996.

[17] Wylie E.B., Streeter V.L., "Fluid Transients in Systems", Prentice Hall, Englewood Cliffs, New Jersey, 1993.

[18] Baltzer R.A., "Column separation accompanying liquid transients in pipes", Jour. Of Basic Engineering, Trans. ASME, series D, vol. 89, n.4, pp. 837-846, 1967.

[19] Ewing D. J. F., "Allowing for free air in water hammer analysis", Proc., 3rd Int. Conf. On Pressure Surges, BHRA, Canterbury, U.K., pp. 127146, 1980. 